\title{
PREDICTING THE SPREAD OF COVID-19 IN IRELAND USING AN AGE-COHORT SEIRD MODEL
}

\author{
SHANE DOYLE* \\ Sponsor: Dr. Petri T Piiroinen ${ }^{\dagger}$
}

\begin{abstract}
The spread of the novel coronavirus SARS-CoV-19 throughout a population can be modelled through the use of compartment models. Here we will use age-cohort separation to design a system of ordinary differential equations, which will be solved with numerical methods in order to model the spread of the virus in Ireland by age-cohort. From here we analyse policy decisions made by the Irish Government throughout the COVID-19 pandemic in early 2020 in terms of their effect on differently aged people within the population. Simulations are generated of alternative policies that could be enacted in the future, with the aim of analysing the effectiveness of policies such as lockdown and cocooning. The results of this analysis indicate that a reduction in social interaction is a major driving force in the suppression of new infections and that reducing the contacts of vulnerable members of the population leads to a slower rate of increase in infections for the population at large. The testing for the model is done by varying the level of social interaction within the population over a 160 day interval from February $29^{\text {th }}, 2020$ until August $7^{\text {th }}$, 2020, with all projections past this date based on assumptions made relating to future levels of social interaction resulting from future policies.
\end{abstract}

Key words. Population Dynamics, Compartmental Modelling, Systems of ODEs, Policy Analysis, COVID-19

1. Introduction. COVID-19 is an infectious disease caused by Severe Acute Respiratory Syndrome Novel Coronavirus 2 (SARS-CoV-2), which is known to cause respiratory infections similar to other diseases caused by coronaviruses such as Middle East Respiratory Syndrome (MERS) and Severe Acute Respiratory Syndrome (SARS)[16] and is known to commonly cause symptoms such as fever, dry cough and tiredness, targeting the infected persons respiratory functions. Around 1 out of every 5 people who gets COVID-19 becomes seriously ill and develops difficulty breathing. Older people and those with underlying medical problems like high blood pressure, heart and lung problems, diabetes or cancer are at a higher risk of developing serious illness. The first verified case occurred in December 2019 in Wuhan, China and has resulted in the ongoing pandemic[19]. It is also very easily transmissable through close contact as the disease spreads primarily from person to person through small droplets from the nose or mouth, which are expelled when a person with COVID-19 coughs, sneezes or speaks. These droplets are relatively heavy, do not travel far and quickly sink to the ground. People can catch COVID-19 if they breathe in these droplets from a person infected with the virus. In the first 6 months of the pandemic we have seen a number of responses using various different policies such as full lockdown, partial lockdown and herd-immunity strategies across many different countries with varying levels of effectiveness.

While the COVID-19 pandemic is a global issue with far-reaching effects, this paper will focus primarily on the progression of the pandemic throughout Ireland, along with the policy that has been implemented to combat the spread of the virus, with support from international data in order to produce an accurate model. In Ireland, a national lockdown was implemented early on in order to reduce social contacts within

\footnotetext{
${ }^{*}$ School of Mathematics, Statistics and Applied Mathematics, National University of Ireland, Galway, Ireland s.doyle31@nuigalway.ie

${ }^{\dagger}$ Division of Dynamics, Department of Mechanics and Maritime Sciences, Chalmers University of Technology, 41296 Gothenburg, Sweden petri.piiroinen@chalmers.se
} 
the population and to delay the spread of the virus. Prior to the lockdown, schools and universities were closed - reducing social contacts for the younger members of the population. Similarly, a policy of "cocooning" was introduced within which elderly members of the population and those with underlying issues causing them to be considered more at-risk were asked to self-isolate for the duration of the pandemic in order to further reduce their social contacts and hence their risk of infection as they would be more adversely affected by the disease than others[13].

In light of Ireland's age-targeted strategies, such as cocooning and school closures, we have chosen to model the spread of COVID-19 in Ireland with the use of an agecohort model, in order to more accurately see the effect of the disease on differently aged members of the population and evaluate policies implemented throughout the pandemic along with the impact of potential future strategies on the diseases spread throughout the Irish population. An age-cohort model is developed, as opposed to a traditional population-level model as used by the Irish government[9] for their policy decisions. An age-cohort model has certain advantages over population-level models as it allows for the population to be broken down and targeted strategies such as cocooning can be modelled more accurately in order to see the effect of policy changes on the targeted age-cohort as opposed to the population as a whole. Within the model it is necessary to account for many parameters that can influence its outcome, which can be thought of as either biological or non-biological, for the sake of categorisation. Biological parameters, such as the incubation and infectious periods of the disease, are assumed to vary from person to person due to differing physiologies among people and are thus implemented as random variables to give a range of potential outcomes within the results of the model. The mortality rate of the disease is treated as a probability of death following the end of the infectious period. This is done using data relating to case fatality rates (CFR)[8] in Ireland in order to infer a probability by Cohort. Interaction within the population is thought of as a non-biological parameter relating to social contacts between infectious and susceptible members of the population effectively dictating new infections. This parameter can be assumed to change as different policies are implemented to alter the spread of the virus and is thus the main parameter used for policy analysis and projections for the future within this model. In terms of policy analysis, a variety of scenarios will be examined in order to determine the effectiveness of implemented policy and to see if there are any other viable alternatives that could have been implemented from the perspective of the model.

The remainder of this paper will be structured as follows. In Section 2 the data required to build the model is explored along with a discussion of the importance of the gathered data to the model. In Section 3 we will first introduce a population-level SEIRD model before progressing to the development of an age-cohort SEIRD model to be used for modelling COVID-19 throughout the remainder of this paper. In Section 4 the methodology for policy analysis is developed further by first establishing initial conditions and deriving parameters from real-world data, leading into a discussion of how the model was implemented within Python along with the method used to analyse alternative policy decisions. In Section 5 the suggested results of the model are explored involving both policy analysis of past policy decisions and potential policy decisions that could have been implemented instead leading onto an analysis of projections for the future under different potential policies. Following this, in Section 6 a conclusion is reached as to the effectiveness of the policies implemented up until this point along with a discussion relating to the most effective future path to take in terms of future infections and deaths. 
2. Data. In this section we will discuss the data used within the model along with the rationale behind the sourcing of this data. Throughout the model we will use known estimates for biological parameters and estimate the effect of nonpharmaceutical interventions by experimentally varying the level of interaction within the population as described later in Section 4.1.1. This is done using daily infection data for Ireland as will now be discussed.

In the course of building this model, reported infections and deaths are used as a basis to generate parameters to best describe the progress of the disease and fit relevant curves to reported cases and deaths. Data relating to new cases by agecohort was obtained from Ordnance Survey Ireland's COVID Statistics Profile[15] from which the daily new infections for the Irish population can be derived (see the data plot shown in Figure 1). It should be noted however that there is a noticeable outlier in this data in mid-April relating to a backlog of cases reported on this day, so this outlier is a result of deficiencies in testing as opposed to a sudden increase in actual cases.

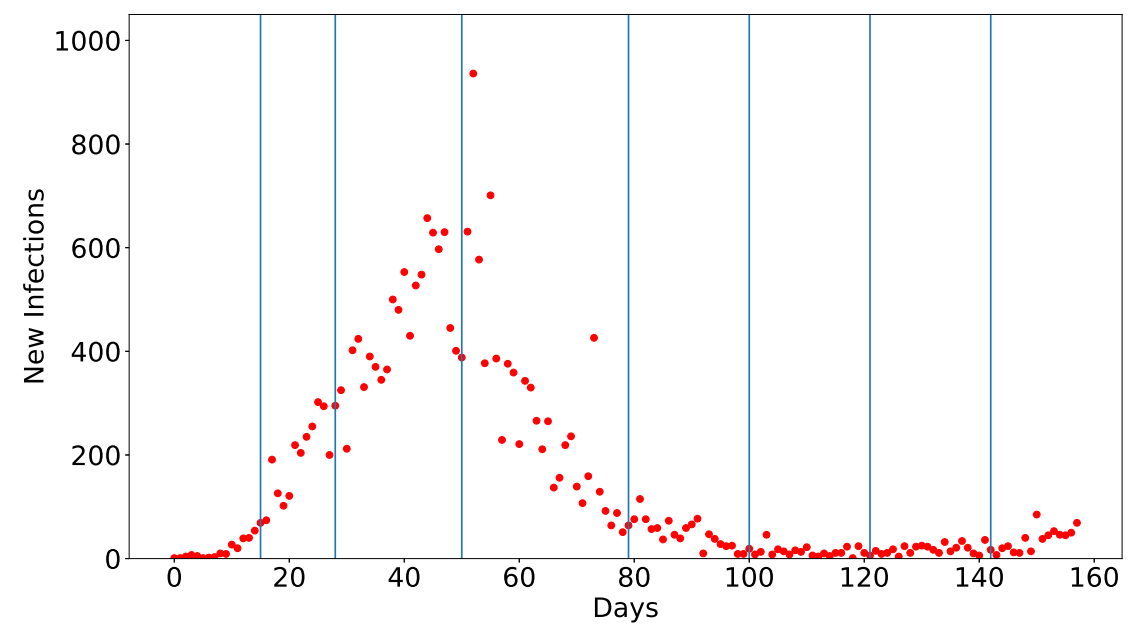

FIG. 1. Daily new infections of the Irish population over 160 days from February $29^{\text {th }}$ sourced from Ordnance Survey Ireland[15] with period subdivisions as highlighted in Figure 5.

The Case Fatality Rate (CFR) of COVID-19 was obtained from the daily mortality data of COVID-19 in Ireland compiled by the Health Protection Surveillance Centre (HPSC) for the National Public Health Emergency Team (NPHET)[8]. This data will be used to infer a probability of death by Cohort, which will be discussed in further detail later in Section 4.1.7.

The population of Ireland and the associated age-cohorts were obtained from data from the 2016 Irish Census available from the Central Statistics Office (CSO)[3], which gave the population breakdown as seen in Table 1. This data will allow us to have Cohorts as an accurate proportion of the population and to more effectively model the spread of the virus by Cohort.

Data on the Effective Reproductive Number $\left(R_{t}\right)$ of the disease, which will be explained in further detail in Section 3.3, was acquired from estimates of $R_{t}$ released 


\begin{tabular}{c|l|c|c} 
Cohort & Age Group & Population Size & Percentage \\
\hline 1 & Persons aged 0-24 & 1583004 & $33.24 \%$ \\
2 & Persons aged 25-64 & 2541294 & $53.37 \%$ \\
3 & Persons aged 65+ & 637567 & $13.39 \%$
\end{tabular}

Irish Population Breakdown (Census 2016)[3] with population divided into Cohorts by age.

by the Irish Epidemiology Modelling Advisory Group (IEMAG)[11] as seen in Table 2 up until the 21 May 2020, with period subdivisions from 1 - 5 representing what IEMAG defines as "Lockdown Phases" including self isolating with symptoms, social distancing, school closures, cancellation of public events, early intervention and lockdown, respectively. The values shown in Table 2 were calculated by IEMAG using the methodology of Imperial College COVID Response Team[11][6]. After this point the

\begin{tabular}{|c|c|c|c|c|c|c|}
\hline & \multicolumn{5}{|c|}{ IEMAG Lockdown Phases } \\
\hline & & 1 & 2 & 3 & 4 & 5 \\
\hline \multirow{5}{*}{ 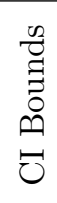 } & $9.5 \%$ & 5.13 & 4.93 & 4.60 & 1.53 & 0.72 \\
\hline & $75 \%$ & 4.75 & 4.40 & 4.01 & 1.36 & 0.66 \\
\hline & $50 \%$ & 4.31 & 4.15 & 3.68 & 1.29 & 0.63 \\
\hline & $25 \%$ & 4.05 & 3.89 & 3.36 & 1.21 & 0.61 \\
\hline & $2.5 \%$ & 3.62 & 3.47 & 2.72 & 1.06 & 0.56 \\
\hline
\end{tabular}

Estimates of $R_{t}$ provided by IEMAG[11] over different "Lockdown Phases" with limits to relevant confidence intervals (CI) until May $20^{\text {th }}$ after which data from NPHET Press briefings[14] was used to construct confidence intervals which are displayed in Figure 8 for comparison against the model.

values were obtained from reports given in NPHET's daily press briefings and from their published meeting minutes[14]. The values and confidence intervals provided are linearly interpolated over time, and will function as a check to ensure that the model is parameterised correctly. This process can be seen more clearly in Figure 8.

3. Model. In this section we will discuss how the age-cohort model was developed by first examining an SEIRD population-level compartmental model, before further developing a system of ordinary differential equations (ODEs) to describe an age-cohort model, along with the reasoning behind this development.

3.1. Population Model. The SEIRD model is an extension of a basic SIR mode as developed by Kermack and Mckendrick in 1927[12]. It is implemented by subdividing a population of size $N$ into Susceptible, Exposed, Infectious, Recovered and Dead Compartments referred to as $S=S(t), E=E(t), I=I(t), R=R(t)$ and $D=D(t)$ respectively, for $t \geq 0$. We consider the actively mixing population $N(t)=S(t)+E(t)+I(t)+R(t)$ with $\frac{d N}{d t}=-\frac{d D}{d t}$. This can be further developed by considering the infectious members of the population contained in $I$ letting this be represented by compartments for presymptomatic, asymptomatic and symptomatic infectious people denoted by $I_{p}=I_{p}(t), I_{a}=I_{a}(t)$ and $I_{s}=I_{s}(t)$, respectively, such that $I=I_{p}+I_{a}+I_{s}$. Movements between these compartments are highlighted in Figure 3. This developed model incorporating various categories of symptoms in infectious members of the population would thus have an actively mixing population given by $N(t)=S(t)+E(t)+I_{p}(t)+I_{a}(t)+I_{s}(t)+R(t)$ with $\frac{d N}{d t}=-\frac{d D}{d t}$ as before. The 
rates of movement between each compartment are given by an interaction coefficient $\beta \geq 0$, a latent incubation rate $\sigma \geq 0$, a presymptomatic incubation rate $\sigma_{p} \geq 0$, a probability of asymptomatic infection $0 \leq \alpha \leq 1$, a reduction factor for infection when symptoms are not present $0 \leq h \leq 1$, a recovery coefficient $\gamma \geq 0$, and a mortality probability $0 \leq \mu \leq 1$, the derivation of which will be discussed further in Section 4.1. This version of the SEIRD model allows for a potentially lethal disease to be modelled through the inclusion of the $D$ compartment, while also allowing for different rates of transmission depending on if symptoms are present by considering the $I_{p}, I_{a}$ and $I_{s}$ compartments as previously described. The movements between compartments is then given by the following system of ODEs

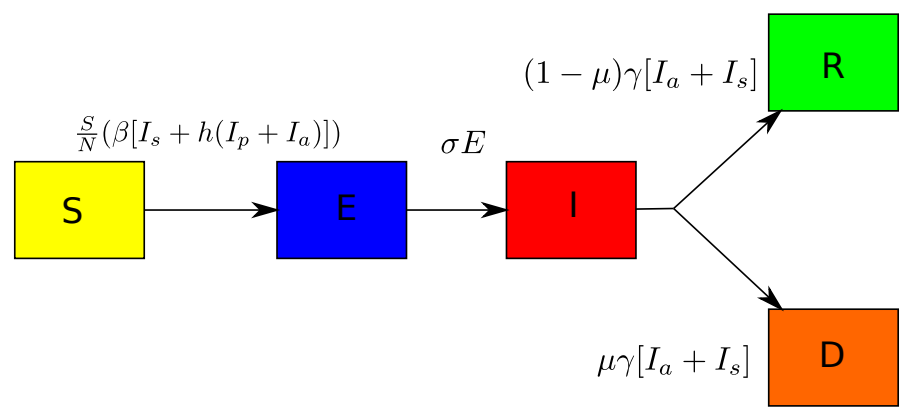

FIG. 2. A population-level SEIRD Model for a lethal disease with a non-infectious incubation period

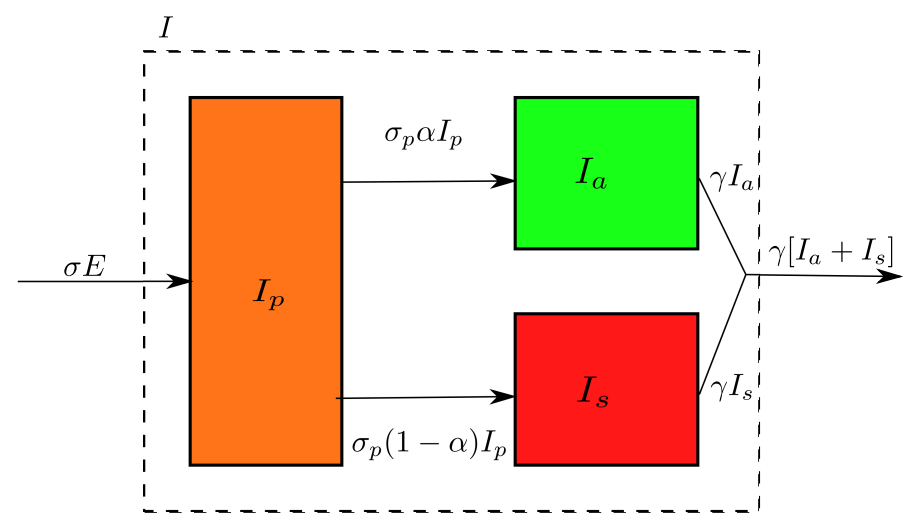

FIG. 3. Actively infectious members of the population incorporating presymptomatic, asymptomatic and symptomatic cases. 


$$
\begin{aligned}
\frac{d S}{d t} & =-\frac{S}{N} \beta\left(I_{s}+h\left(I_{a}+I_{p}\right)\right), \\
\frac{d E}{d t} & =\frac{S}{N} \beta\left(I_{s}+h\left(I_{a}+I_{p}\right)\right)-\sigma E, \\
\frac{d I_{p}}{d t} & =\sigma E-\sigma_{p} I_{p}, \\
\frac{d I_{a}}{d t} & =\alpha \sigma_{p} I_{p}-\gamma I_{a} \\
\frac{d I_{s}}{d t} & =(1-\alpha) \sigma_{p} I_{p}-\gamma I_{s}, \\
\frac{d R}{d t} & =\gamma(1-\mu)\left(I_{a}+I_{s}\right), \\
\frac{d D}{d t} & =\gamma \mu\left(I_{a}+I_{s}\right),
\end{aligned}
$$

3.2. Age-Cohort Model. The population-level model discussed in Section 3.1 functions well to provide a broad overview of the progress of a disease within a population. However, within these models it is assumed that there is a homogeneously dispersed population with equal levels of interaction between all Infectious and Susceptible people throughout the population. For example, an infectious 97 year old is assumed to interact with a susceptible 7 year old at the same rate at which that 7 year old might interact with other infectious 7 year olds. For this reason, an agecohort variant of the above models can be developed to allow for a breaking down of the population into $k \in \mathbb{N}$ age-cohorts with a separate interaction coefficient $\beta_{i j}$ between susceptible people from Cohort $i$ and infectious people in Cohort $j$ which can be represented in a $k \times k$ matrix $\beta$,

$$
\beta(t)=\left(\begin{array}{ccc}
\beta_{11}(t) & \cdots & \beta_{1 k}(t) \\
\vdots & \ddots & \vdots \\
\beta_{k 1}(t) & \cdots & \beta_{k k}(t)
\end{array}\right)
$$

The model is then extended to $k$ classical SEIRD models in parallel, as seen in Figure 4. Each Cohort $i=1, \ldots, k$ is assumed to be of size $N_{i}$ such that $\sum_{i=1}^{k} N_{i}=N$. Accounting for interactions between all susceptible and infectious people in Cohorts $i$ and $j$, respectively, as highlighted in Figure 4 gives us the system 


$$
\begin{aligned}
\frac{d S_{i}}{d t} & =-\frac{S_{i}}{N_{i}}\left(\sum_{j=1}^{k} \beta_{i j}(t)\left[I_{s j}+h\left(I_{p j}+I_{a j}\right)\right]\right) \\
\frac{d E_{i}}{d t} & =\frac{S_{i}}{N_{i}}\left(\sum_{j=1}^{k} \beta_{i j}(t)\left[I_{s j}+h\left(I_{p j}+I_{a j}\right)\right]\right)-\sigma E_{i}, \\
\frac{d I_{p i}}{d t} & =\sigma E_{i}-\sigma_{p} I_{p i} \\
\frac{d I_{a i}}{d t} & =\alpha \sigma_{p} I_{p i}-\gamma I_{a i} \\
\frac{d I_{s i}}{d t} & =(1-\alpha) \sigma_{p} I_{p i}-\gamma I_{s i} \\
\frac{d R_{i}}{d t} & =\gamma\left(1-\mu_{i}\right)\left(I_{a i}+I_{s i}\right), \\
\frac{d D_{i}}{d t} & =\gamma \mu_{i}\left(I_{a i}+I_{s i}\right),
\end{aligned}
$$

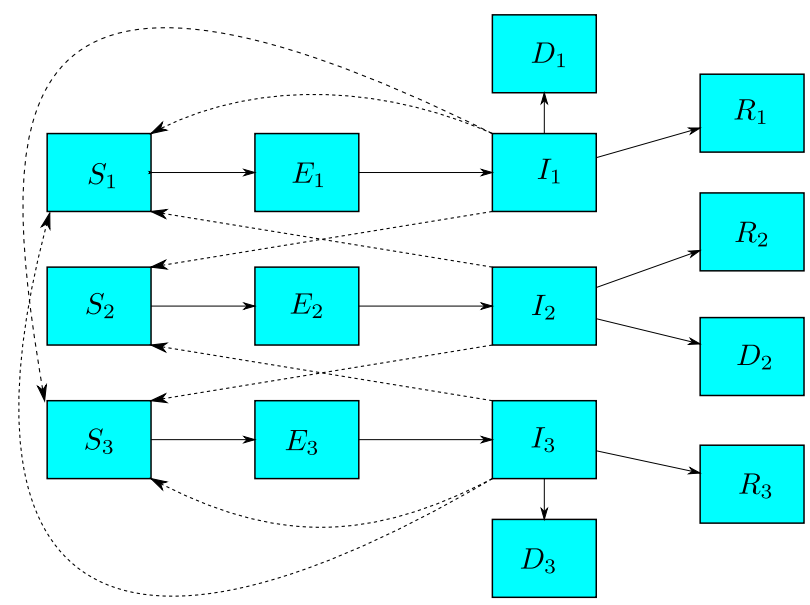

FIG. 4. An age-cohort model for $k=3$ Cohorts functioning as 3 classical SEIRD models running in parallel with interaction between Susceptible and Infectious people from each Cohort highlighted by dashed feedback loops. The $I_{i}$ compartments seen here behave as shown in Figure 3.

3.3. Reproductive Number. The basic reproductive number of an infectious disease $R_{0}$ can be thought of as the number of secondary infections generated from a single initial infection. An $R_{0}>1$ indicates that the number of infections over time will increase exponentially, while an $R_{0}<1$ indicates that the disease will die out over time as its secondary infections will not sustain its growth over the long term.

Following the notation of Heffernan, Smith and Wahl[7], the effective reproductive number $\left(R_{t}\right)$ is given as the spectral radius of the "next generation operator" $F V^{-1}$, where on a population level, instead of the distinct compartment given to each of the given $k \in \mathbb{N}$ Cohorts, the population can be viewed as consisting of 5 compartments given by

$$
x_{1}=\sum_{i=1}^{k} S_{i}, \quad x_{2}=\sum_{i=1}^{k} E_{i}, \quad x_{3}=\sum_{i=1}^{k} I_{i}, \quad x_{4}=\sum_{i=1}^{k} R_{i}, \quad x_{5}=\sum_{i=1}^{k} D_{i} .
$$


It should be noted that in defining the compartment $x_{3}$ in Equation (3.16) we let $I_{i}=I_{a i}+I_{p i}+I_{s i}$ denote all actively infectious members of cohort $i$, which we will use below for brevity. It can be seen that out of the $n=5$ compartments, $m=2$ of these can be considered to be infected which in turn can be seen as the vector of relevant dynamic variables $x=\left(x_{2}, x_{3}\right)$. Denoting $N=\sum_{i=1}^{5} x_{i}$ we can define $F_{i}(N)$ as the rate of appearance of new infections in compartment $i$ and letting $V_{i}(N)$ denote the net rate of transfer from compartment $i$ by all other means. This thus gives $F_{i}(N)-V_{i}(N)$ to be the rate of change of compartment $i$. The next-generation operator can thus be defined as the matrix of partial derivatives of $F_{i}$ and $V_{i}, i=2,3$ and $j=2,3$, with $x_{0}$ being a point where the $m$ infected compartments are empty with $x_{2}=0$ and $x_{3}=0$. The point $x_{0}$ is known as the disease-free equilibrium[7] and $F$ and $V$ can be defined as

$$
F=\left[\frac{\partial F_{i}\left(x_{0}\right)}{\partial x_{j}}\right], \quad V=\left[\frac{\partial V_{i}\left(x_{0}\right)}{\partial x_{j}}\right], \quad i, j=2,3
$$

In the case of the age-cohort SEIRD model described in Section 3.2 containing $k=3$ age cohorts the matrices for $F$ and $V$ can be given as

$$
F=\left(\begin{array}{cc}
0 & \sum_{i=1}^{3} \sum_{j=1}^{3} \beta_{i j} \\
0 & 0
\end{array}\right), \quad V=\left(\begin{array}{cc}
\sigma & 0 \\
-\sigma & \sum_{i=1}^{3} \gamma_{i}
\end{array}\right)
$$

The matrix $F V^{-1}$ can thus be calculated from the given matrices $F$ and $V$, with the dominant eigenvalue taken as the value for $R_{0}$, which is given by

$$
R_{0}=\frac{\sum_{i=1}^{3} \sum_{j=1}^{3} \beta_{i j}}{\sum_{i=1}^{3} \gamma_{i}},
$$

the details of this can be seen in Appendix A. From this value for $R_{0}$ we can now account for varying levels of infection over time and partial immunity of the population as people leave the Susceptible compartment by calculating the effective reproductive number $R_{t}[5]$. This is done by letting $\beta_{i j}=\beta_{i j}(t)$ evolve over time and scaling $R_{0}$ to the proportion of Susceptible people within the population, thus creating an effective reproductive number $R_{t}$ as a time-dependent function, such that

$$
R_{t}=\frac{S(t)}{N(t)} R_{0}=\frac{S(t)}{N(t)} \frac{\sum_{i=1}^{3} \sum_{j=1}^{3} \beta_{i j}}{\sum_{i=1}^{3} \gamma_{i}} .
$$

It is important to note that while the reproductive number does give a general overview of the projected growth of a disease in the early stages of an epidemic, it is not a summary value that encompasses the entire disease and should be used together with other indicators to give an accurate picture of the disease as it can be easily skewed by a small initial number of infected people and clusters. 
4. Methodology. In this section we will discuss the methodology used to develop the model and to produce the results seen in Section 5 by discussing the generation of parameters and the methods used to carry out simulations in Python. In order to implement this model and run simulations, the age-cohort model developed in Section 3.2 is established for $k=3$ Cohorts, these being those aged $0-24,25$ 64 and $65+$, or in other words the young, adult and elderly Cohorts, respectively. The size of each of these Cohorts can be seen in Table 1 and initial conditions are established such that $t_{0}=0$ on the $29^{\text {th }}$ February when the Republic of Ireland reported its first verified case, the initial population is taken as $N=4937786$ with the breakdown of the population such that the populations of Cohorts 1, 2 and 3 where $N_{1}=1641320, N_{2}=2635296$ and $N_{3}=661170$, respectively, as seen in Table 1 . The population of each cohort is taken to be strictly decreasing with the death rate such that $\frac{d N_{i}}{d t}=-\frac{d D_{i}}{d t}$. In the following analysis we will consider the grouping of actively infectious people in cohort $i, I_{i}=I_{p i}+I_{a i}+I_{s i}$ for clarity when comparing the model with data in Section 5. The initial Irish infection is assumed to be a member of the adult population of Cohort 2 presenting with symptoms with a subsequent member of Cohort 2 being exposed such that $I_{s 2}(0)=I_{2}(0)=1$ and $E_{2}(0)=1$ with no infectious or exposed people in the remaining compartments with $I_{1}(0)=I_{3}(0)=0$ and $E_{1}(0)=E_{3}(0)=0$. Initially there are no recovered or dead members of the population and thus $R_{1}(0)=R_{2}(0)=R_{3}(0)=0$ and $D_{1}(0)=D_{2}(0)=D_{3}(0)=0$. Following this, the size of the Susceptible compartment is taken as the residual of all other compartments at any given point in time $t$ such that $S_{i}(t)=N_{i}(t)-E_{i}(t)-I_{i}(t)-R_{i}(t)$, $i=1,2,3$.

The model is run over 160 days from the first verified Irish case which was notified on $29 / 2 / 2020$ deemed to be $t_{0}=0$ as mentioned above, until $7 / 8 / 2020$ the final time point $t_{8}=160$. Changes in policy such as the implementation of lockdown strategies, or the phases of reopening from lockdown implemented by the Irish government, are seen as the start of a new period, with a different level of social interaction implemented for each period in order to reflect behaviour within that time period, as shown in Figure 5 with $n=8$ periods within the model separated by the following dates in 2020;

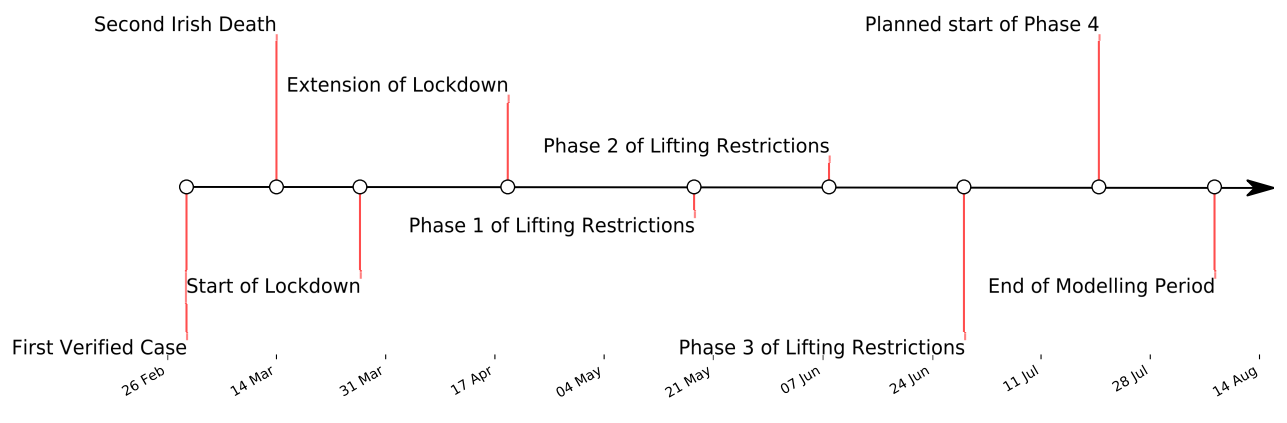

FIG. 5. Irish timeline of policy changes with relevant dates when social interaction is deemed to change marking the beginning of one of the $n=8$ periods used within the model highlighted. 
Feb $29^{\text {th }}\left(t_{0}=0\right)$ : The first verified Irish case.

Mar $14^{\text {th }}\left(t_{1}=15\right)$ : The second Irish death from which a "cautious phase" is assumed to begin, where social interaction is reduced. Schools are also closed and a travel restriction of $5 \mathrm{~km}$ for non-essential journeys is put in place.

Mar $27^{\text {th }}\left(t_{2}=28\right)$ : The start of the Irish lockdown within which the elderly are asked to cocoon, reducing their social contacts, along with the cessation of all labour, aside from that deemed to be "essential" by the Irish government.

Apr $19^{\text {th }}\left(t_{3}=50\right)$ : The extension of the Irish lockdown.

May $18^{\text {th }}\left(t_{4}=79\right)$ : Start of phase 1 of the Irish reopening, where outdoor-based retailers are allowed to reopen.

Jun $8^{\text {th }}\left(t_{5}=100\right)$ : Start of phase 2 of Irish reopening, where visits between households are permitted along with the further reopening of businesses and the lifting of travel restrictions.

Jun $26^{\text {th }}\left(t_{6}=121\right)$ : Start of phase 3 of the Irish reopening, where most retailers are permitted to return to work along with café's, restaurants and pubs serving food, sporting activities are also permitted to resume along with the reopening of schools for the upcoming academic year.

Jul $20^{\text {th }}\left(t_{7}=142\right)$; Planned date for Phase 4 of reopening, which would have entailed a full economic reopening with only the support of public health advice, which was then delayed beyond the scope of the modelling period.

Aug $7^{\text {th }}\left(t_{8}=160\right)$ : End of the modelling period.

4.1. Parameters. The model relies on a number of parameters, as seen in Equations (3.9) - (3.15), which reflect both the behaviour of people within a population and the diseases effect on people. The biological parameters within the model that cannot be varied with policy are derived from the data examined in Section 2, with the fitting of parameters carried out using the Irish reported infection data shown in Figure 1. This establishment of parameters is important in order to achieve an accurate representation of the models path thus far and hence allow for reliable policy analysis later in Section 5. We will now establish the necessary parameters in Sections 4.1.1 - 4.1.7 with a summary of these values available in Table 4

4.1.1. Interaction and Policy. Interaction between susceptible and infectious people can be shown using the $\beta_{i j}$ parameter as discussed in Section 3.2. This parameter can be thought of as the number of social contacts leading to an infection between a Susceptible person in Cohort $i$ and an Infectious person in Cohort $j$ per day. As social distancing and lockdown policies are implemented throughout the course of the disease's outbreak, as was the case in Ireland. The rate of interaction between these groups can be assumed to change over time as new policies are implemented, therefore we define $\beta_{i j}(t)$ as a piecewise constant function over time taking on a constant value $b_{i j}^{(\kappa)}$ within a period $\kappa=\left[\begin{array}{ll}t_{\kappa}, & t_{\kappa+1}\end{array}\right)$ in order to reflect interaction between susceptible people in Cohort $i$ and infectious people in Cohort $j$ within a period $\kappa$ given by the time points described in Section 4,

$$
\beta(t)=\left(\begin{array}{lll}
\beta_{11}(t) & \beta_{12}(t) & \beta_{13}(t) \\
\beta_{21}(t) & \beta_{22}(t) & \beta_{23}(t) \\
\beta_{31}(t) & \beta_{32}(t) & \beta_{33}(t)
\end{array}\right), \quad \beta_{i j}(t)=\left\{\begin{array}{cc}
b_{i j}^{(0)}, & t_{0} \leq t<t_{1}, \\
\vdots & \vdots \\
b_{i j}^{(\kappa)}, & t_{\kappa} \leq t<t_{\kappa+1}, \\
\vdots & \vdots \\
b_{i j}^{(n-1)}, & t_{n-1} \leq t<t_{n} .
\end{array}\right.
$$


The estimated values for $b_{i j}^{(\kappa)}$ can be seen in Table 5 for all periods $0 \leq \kappa \leq 7$. These values were chosen in order to capture the overall trajectory of the daily confirmed infection data.

4.1.2. Incubation Rate. The incubation period $L$ of the disease is the period for which a person is infected but is not infectious, the inverse of this produces the rate of incubation $\sigma=\frac{1}{L}$, which is used in Equation (3.10). This is assumed to be a random variable following a Lognormal distribution as suggested by the Parameter Summary Document produced by the Irish IEMAG Modelling Subgroup[10] and thus,

$$
L \sim \operatorname{Ln}(\mu=1.63, \sigma=0.5) .
$$

This produces a distribution as seen in Figure 6(a), allowing for the case that the disease may have a very long incubation period in certain individuals, which can be seen from the right skew given by the Lognormal distribution.

4.1.3. Presymptomatic Incubation Rate. The presymptomatic incubation rate $\sigma_{p}=\frac{1}{P}$ is given by the inverse of the presymptomatic period for an infected person, that is the period for which a person is infectious but does not yet show symptoms. This is assumed to be a constant value of $P=2$ days across all age cohorts in line with a study relating to the transmission dynamics of COVID-19 carried out by Cevic et al.[4].

$$
\sigma_{p}=\frac{1}{2}
$$

4.1.4. Probability of Asymptomatic Infection. The probability of asymptomatic infection $\alpha$ is the probability that following the presymptomatic period of infection the infected person will not develop symptoms. This is assumed to be constant across all age-cohorts and is derived from a meta-analysis of Asymptomatic SARS-CoV-2 infections[2] where from an analysis of 94 studies the overall estimate of the proportion of people who become infected with SARS-CoV-2 and remain asymptomatic throughout infection was $20 \%$ (95\% CI 17-25).

$$
\alpha=0.2 .
$$

This dictates movement from $I_{p}$ to $I_{a}$ with $(1-\alpha) I_{p}$ moving from $I_{p}$ to $I_{s}$ presenting symptoms.

4.1.5. Infectivity Reduction Factor. The infectivity reduction factor $h$ is a multiplicative factor that reduces the transmission rate between between Susceptible people contained in $S$ and Infectious people that do not present symptoms contained in $I_{p}$ and $I_{a}$. This is taken as the arithmetic mean of the transmission rate between asymptomatic and susceptible individuals derived from a number of studies used in an analysis of Symptomatic and Asymptomatic Infection by Arcede et al.[1]. From this we established $h=0.78$. As both presymptomatic and asymptomatic cases do not present with symptoms this reduction factor is assumed to hold for transmission from $I_{p}$ and $I_{a}$ within the model.

4.1.6. Recovery Rate. The infectious period $D$ is also known to vary from person to person and is taken from a uniform distribution in the interval [3,9] within the model, with the rate of recovery taken as the inverse of this infectious period $\gamma=\frac{1}{D}$, which is used in Equations (3.12) - (3.15). We can hence see that it follows the distribution $D \sim \mathrm{U}(3,9)$. This is taken from the inter-quartile range of hospital 
stay outside of China according to a study on length of hospital stay from the London School of Hygiene and Tropical Medicine[17], as discussed in Section 2. This is similarly taken as a random variable for simulations as this varies on a case by case basis depending on physiology. A Gamma/Weibull distribution was suggested by the London School of Hygiene and Tropical Medicine as it takes into account fringe cases that spend a very long period of time in hospital due to the right skew from these distributions. However a uniform distribution is used here as seen in Figure 6(b) as a simplifying assumption.

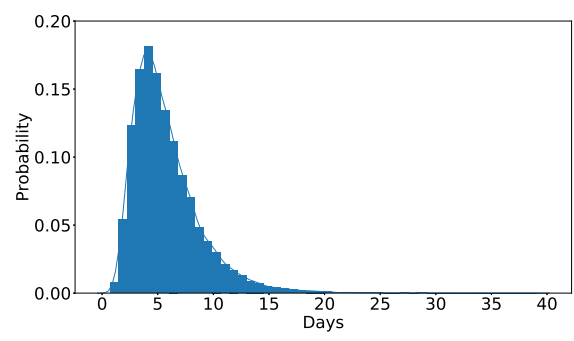

(a) Incubation Periods

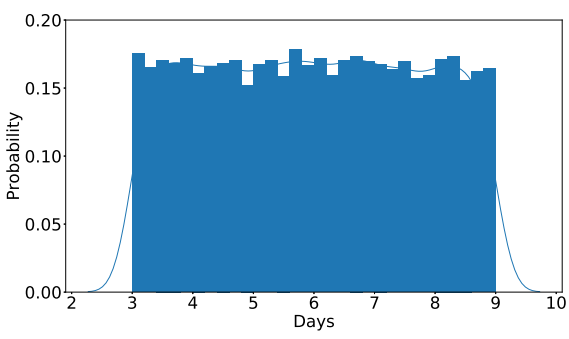

(b) Infectious Periods

FIG. 6. Distribution of incubation[10] and infectious[17] periods given as a lognormal and uniform distribution, respectively, according to the parameters as discussed in Section 4.1.2 and 4.1.6

4.1.7. Mortality Probability. Mortality is given as a probability of death at the end of the infectious period. This is implemented as the mean of the reported CFR by age-cohort within Ireland over 10 days from $21 / 7 / 2020$ to $31 / 7 / 2020$. This was sourced from the daily Epidemiology of COVID-19 in Ireland reports from the HPSC[15], with case statistics updated daily under the assumption that as time progresses and more testing is done, the available sample will increase such that the "true" CFR will be approached by the reported data. The value of this "true" CFR is shown in Table 3 and implemented within the model as the values for $\mu_{i}$ in Equation (3.15).

\begin{tabular}{c|c|c} 
Cohort & Age Group & Death Rate $\left(\mu_{i}\right)$ \\
\hline 1 & Persons aged 0-24 & $3.97 \times 10^{-4}$ \\
2 & Persons aged 25-64 & $6.40 \times 10^{-3}$ \\
3 & Persons aged 65+ & $2.15 \times 10^{-1}$ \\
& \multicolumn{2}{|c|}{ TABLE 3 }
\end{tabular}

Values for probability of death by Cohort ( $\mu_{i}$ for a Cohort $i$ ) inferred from reported CFR from $H P S C[8]$. 


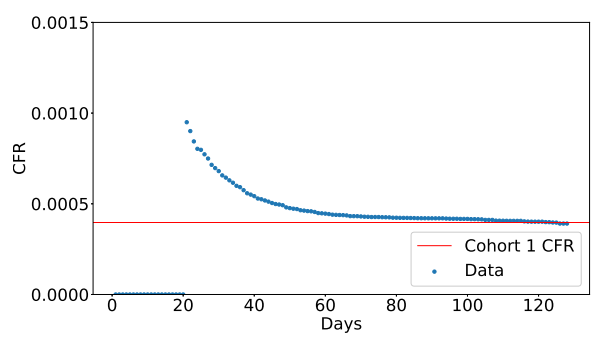

(a) Cohort 1

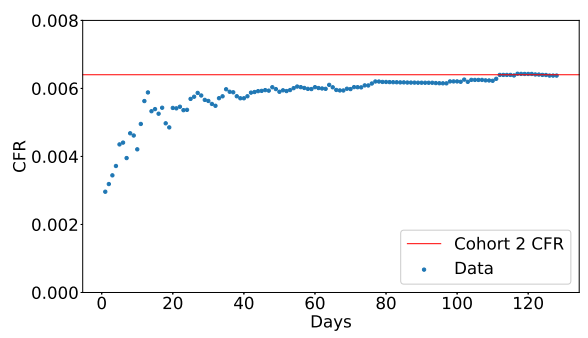

(b) Cohort 2

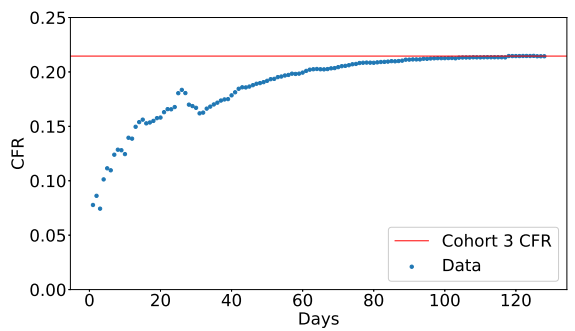

(c) Cohort 3

FIG. 7. Case Fatality Rate (CFR) for Cohorts 1-3 inferred from HPSC Reports[8] by taking the mean of the reported CFR for 10 days from $21^{\text {st }}-31^{\text {st }}$ July.

\begin{tabular}{c|l|c} 
Parameter & \multicolumn{1}{|c}{ Description } & Value \\
\hline$\beta_{i j}$ & Cohort Interaction Coefficient & See Table 5 \\
$\sigma$ & Incubation Rate & $\frac{1}{1.63}$ (mean) \\
$\sigma_{p}$ & Presymptomatic Incubation Rate & 0.5 \\
$\alpha$ & Probability of Asymptomatic Infection & 0.2 \\
$h$ & Infectivity Reduction Factor & 0.78 \\
$\gamma$ & Recovery Rate & $\frac{2}{9}$ (mean) \\
$\mu_{i}$ & Cohort Mortality Probability & See Table 3 \\
\multicolumn{2}{c}{ Summary of parameters described in Sections 4.1.1 - 4.1.7 }
\end{tabular}

4.2. Method for Policy Analysis. In order to carry out policy analysis the model was implemented in Python[18]. A number of Python libraries were used to assist in the development of the model such as:

- numpy was used in order to run multiple simulations using $n$-dimensional numpy arrays as opposed to native Python tools in order to compute with and manipulate the large amounts of data used within the model effectively.

- scipy.integrate was used in order to numerically solve the system of ODEs within the model through the use of the solve_ivp() function which used an RK45 solver to solve the system.

- pandas was used in order to pass real-world data into the model for comparison, parameter-fitting and to carry out analysis of results.

- matplotlib.pyplot was used in order to effectively visualise the data and model output which can be seen in Section 5 . 
In order to carry out simulations and hence policy analysis using these libraries along with real-world data, the model was implemented in Python using the following method:

- The system of ODEs discussed in Section 3.2 was defined as a function within Python with the initial conditions and population sizes discussed earlier on Section 4.

- The incubation and infectious periods discussed in Sections 4.1.2 and 4.1.6 relating to the $\sigma$ and $\gamma$ parameters, respectively, were generated as a list of length 25000 using the numpy.random library according to their lognormal and uniform distributions, respectively. The death rate for each Cohort was implemented using the values seen in Table 3.

- The system of ODEs was then solved numerically 25000 times over the provided randomly generated values for $\sigma$ and $\gamma$ using the solve_ivp() function from the scipy.integrate library to produce a numpy array of resulting values for each Cohort, compartment and set of values for $\sigma$ and $\gamma$.

- This numpy array was then split to produce a range of values for each compartment over time with the $25^{\text {th }}$ and $75^{\text {th }}$ quantiles taken for the infection range produced using the numpy -quantile() function.

- The constant values $b_{i j}^{(\kappa)}$, attributed to $\beta_{i j}(t)$ at a given time $t \in\left[t_{\kappa}, t_{\kappa+1}\right)$ as shown in Equation (4.1), was altered for each Cohort by period such that the curve produced by the data lay within the models output range, with the $50^{\text {th }}$ quantile used as a guide for the reported data.

- The reported value for the effective reproductive number was also used to check that the model output was in line with real-world data, as shown in Figure 8.

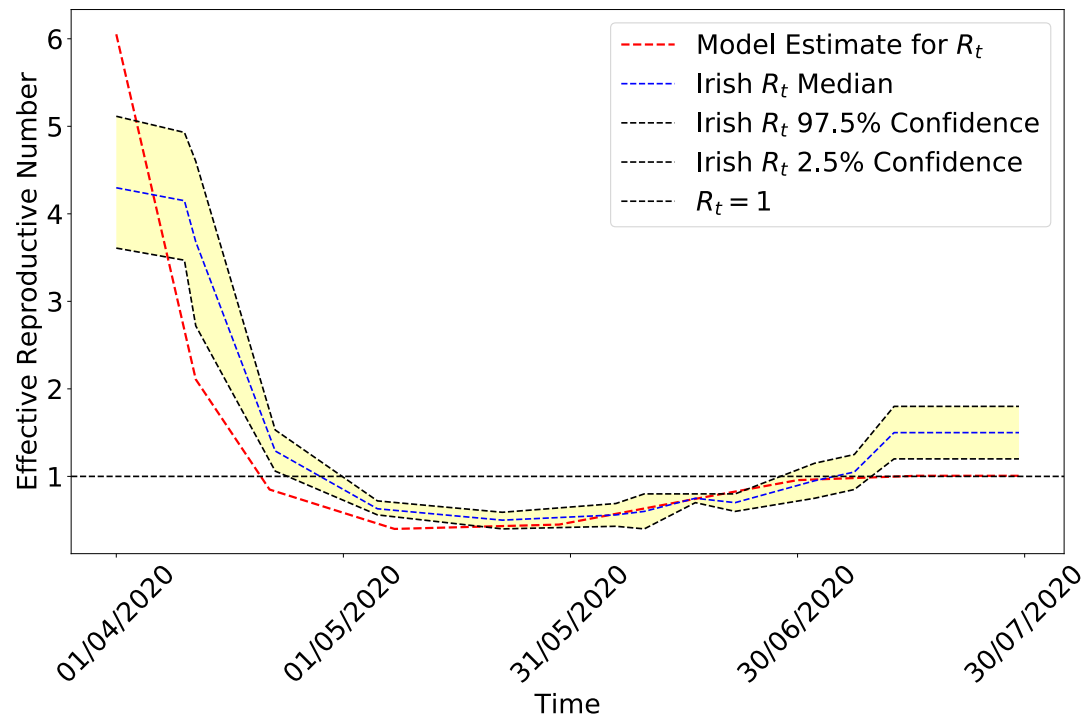

FIG. 8. Model output for the reproductive number $\left(R_{t}\right)$ calculated using the method described in Section 3.3 to calculate a population-level value for $R_{t}$ compared with reported $95 \%$ quantile range given by IEMAG[11] and NPHET[14]. 
In order to carry out policy analysis the originally assigned value of $b_{i j}^{(\kappa)}$ could be altered from the original fitted value in order to reflect a higher or lower level of interaction between susceptible people from Cohort $i$ and infectious people in Cohort $j$ during the period that the time $t$ lies within. This will be discussed in more detail in Section 5.1. Projections for the future were generated by extending the modelling period by including a new time period for all $t \geq 160$, where an assumption relating to the level of social interaction following the end of the modelling period would be made and a projection carried out under the assumption that there is no further policy intervention aside from the assumed policy intervention at the end of the modelling period.

5. Results. With the methodology established in Section 4 we can carry out simulations relating to implemented policy and potential policies in terms of number of infections and the growth rate of these infections experienced over time. We can observe the current course of the virus by incorporating actual past policy decisions, as seen in Figure 9, with a $50 \%$ quantile range produced about the $50^{\text {th }}$ quantile following the constant values assigned for $\beta_{i j}(t)$ described in Table 5 . The data points for confirmed Irish daily infections can be seen to fall along the $50^{\text {th }}$ quantile line in Figure 9(a) representing the population at large. From examining this data it should be noted that, specifically in Figures $9(\mathrm{~d})$ and $9(\mathrm{e})$, there is a notable outlier around day 50 of the model, this relates to a backlog in tested cases, as previously mentioned in Section 2. Upon division into Cohorts and scaling to Cohort population size the model produces curves consistent with Figures $9(\mathrm{~b})-9(\mathrm{~d})$. The data for Cohort 1 can be seen to occur below the $50^{\text {th }}$ quantile line which is likely due to reporting of confirmed cases at a lower rate than the model would suggest that actual cases are occurring, a possible explanation for this might be that younger people are less likely to present with severe symptoms of COVID-19 than older Cohorts[13] and so it is less frequently reported. Cohort 2 returns a better fit about the $50^{\text {th }}$ quantile than Cohort 1, as Cohort 2 contains those aged 25-64, encompassing the majority of the Irish workforce, it can also be seen that the model returns a higher maximum range than the other Cohorts in terms of Cohort size - meaning that a larger proportion of the Cohort 2 population is infected when compared with other Cohorts. This is most likely due to the higher levels of interaction associated with being in the workforce along with the presence of "essential workers" who still presented for work throughout the lockdown. Cohort 3's confirmed cases appear in the higher end of the model range. As the elderly were encouraged to cocoon during lockdown this is reflected by the level of interaction involving Cohort 3 implemented in the model during lockdown. Similarly, due to the more adverse effects associated with COVID19 for older members of the population[13] the confirmed cases for Cohort 3 are more likely to be in line with actual cases than other Cohorts. Taking these factors into account it can be seen that the range associated with Cohort 3 produced by the model is lower than the data might suggest. We can also see the death curve for the population in Figure $9(\mathrm{e})$, the data for which was seen to lie between the $90^{\text {th }}$ and $99^{\text {th }}$ quantiles, with the data peaking at approximately 1500 deaths at the end of the modelling period.

With the current policies implemented as of the end of the modelling period, the model suggests that if no further intervention is made, the virus may re-emerge within the coming months, likely due to a higher level of social interaction in the later stages of reopening. This will be discussed in greater detail in Section 5.2.

From this initial analysis of implemented policies the level of interaction within 


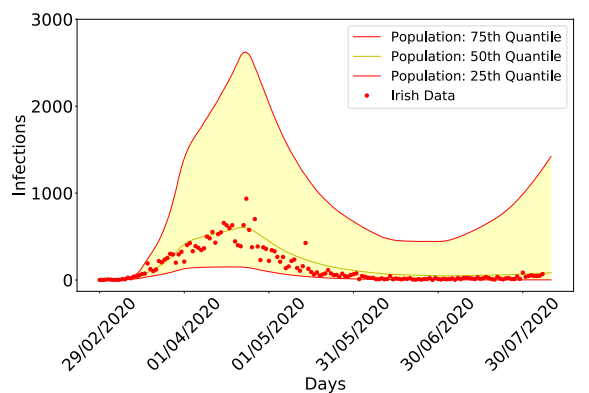

(a) Population

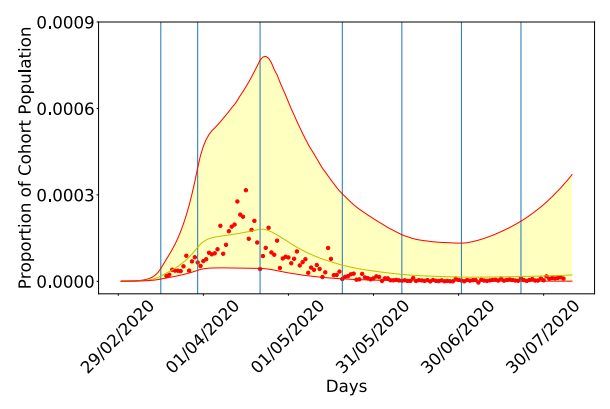

(c) Cohort 2 scaled to Cohort population

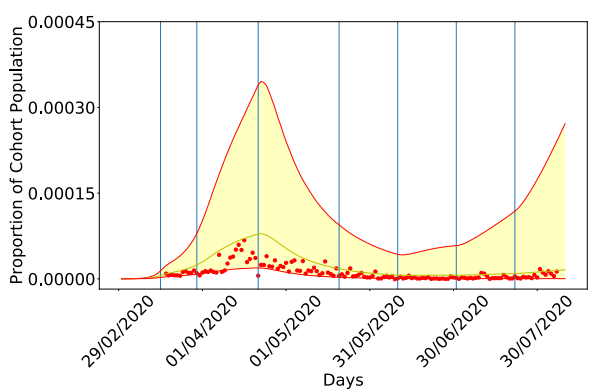

(b) Cohort 1 scaled to Cohort population

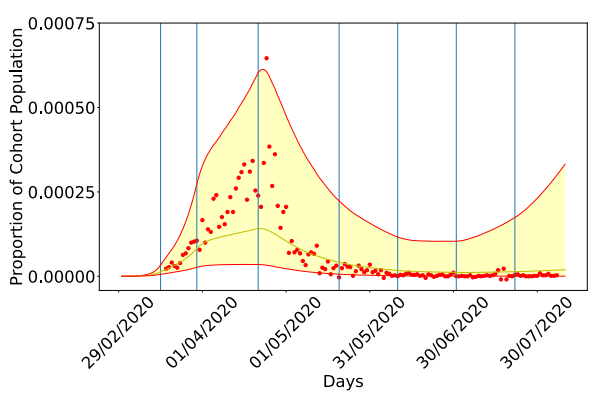

(d) Cohort 3 scaled to Cohort population

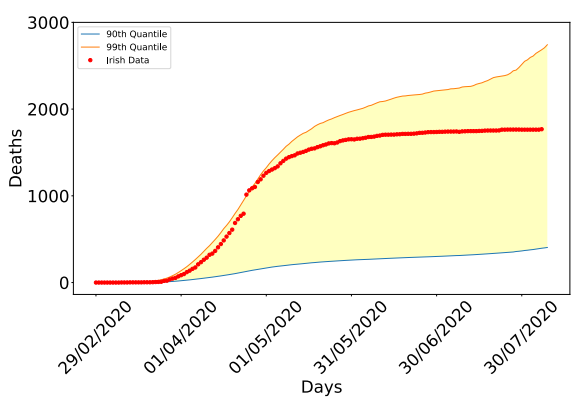

(e) Population Death Curve

FIG. 9. A $50 \%$ quantile range from the $25^{\text {th }}$ to the $75^{\text {th }}$ quantiles for infection curves with a quantile range from the $90^{\text {th }}$ to the $99^{\text {th }}$ quantiles for the death curve, fitted to data of actual lockdown policy used in Ireland.

the model can be altered to reflect different policies that could have and could be enacted in the future. Such simulations will now be discussed in Sections 5.1 and 5.2.

5.1. Policy Analysis. In this section we will carry out an analysis of alternative policies that could have been implemented throughout the modelling period as opposed to the policy timeline shown in Figure 5 leading to the results detailed in Figure 9. This will allow us to evaluate the effectiveness of implemented policies along with how other policy decisions might have impacted the behaviour of the virus throughout the modelling period. The policies that are retrospectively seen to have 


\begin{tabular}{c|cccccccc} 
& $t_{0}$ & $t_{1}$ & $t_{2}$ & $t_{3}$ & $t_{4}$ & $t_{5}$ & $t_{6}$ & $t_{7}$ \\
\hline$\beta_{11}$ & 0.15 & 0.03 & 0.03 & 0.01 & 0.01 & 0.025 & 0.045 & 0.055 \\
$\beta_{12}$ & 0.15 & 0.03 & 0.03 & 0.01 & 0.01 & 0.025 & 0.035 & 0.045 \\
$\beta_{13}$ & 0.1 & 0.03 & 0.03 & 0.01 & 0.01 & 0.015 & 0.025 & 0.035 \\
$\beta_{21}$ & 0.4 & 0.25 & 0.1 & 0.07 & 0.07 & 0.08 & 0.1 & 0.1 \\
$\beta_{22}$ & 0.7 & 0.25 & 0.15 & 0.07 & 0.07 & 0.08 & 0.1 & 0.1 \\
$\beta_{23}$ & 0.65 & 0.25 & 0.03 & 0.01 & 0.04 & 0.07 & 0.08 & 0.08 \\
$\beta_{31}$ & 0.5 & 0.125 & 0.03 & 0.02 & 0.02 & 0.04 & 0.06 & 0.06 \\
$\beta_{32}$ & 0.55 & 0.15 & 0.08 & 0.02 & 0.02 & 0.04 & 0.06 & 0.06 \\
$\beta_{33}$ & 0.6 & 0.15 & 0.03 & 0.02 & 0.02 & 0.05 & 0.07 & 0.07
\end{tabular}

Evolution of $\beta_{i j}$ between Cohorts over the modelling period, with $\beta_{i j}(t)$ assigned a constant value $b_{i j}^{(\kappa)}$ for a time value $t \in\left[t_{\kappa}, t_{\kappa+1}\right)$, such that the period given by the interval $\left[t_{\kappa}, t_{\kappa+1}\right)$ is known as period $\kappa$. Values were estimated to roughly fit confirmed infection data points around the $50^{\text {th }}$ quantile from multiple simulations.

been quite effective can then be brought forward and examined in Section 5.2 in terms of projections for the future as potential policy.

5.1.1. No Lockdown. The use of a no lockdown simulation serves to illustrate the unhindered course of the virus if no action was taken and can indicate if policy intervention was necessary. This scenario can be simulated by assigning the value of all $\beta_{i j}(t)$ to a constant value for the duration of the modelling period, namely, their value at $t=0$ from the initial analysis $b_{i j}^{(0)}$ such that $\beta_{i j}(t)=b_{i j}^{(0)}$ for all $t \geq 0$. It can be

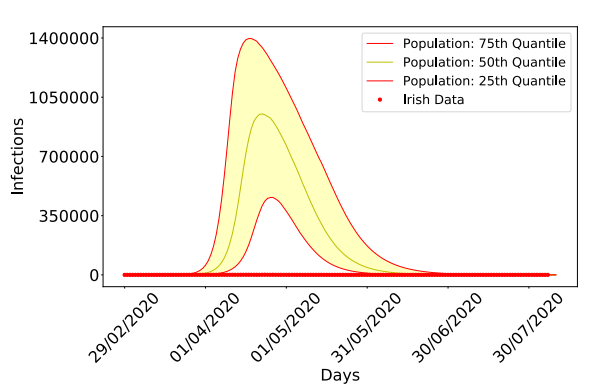

(a) Infection Curve

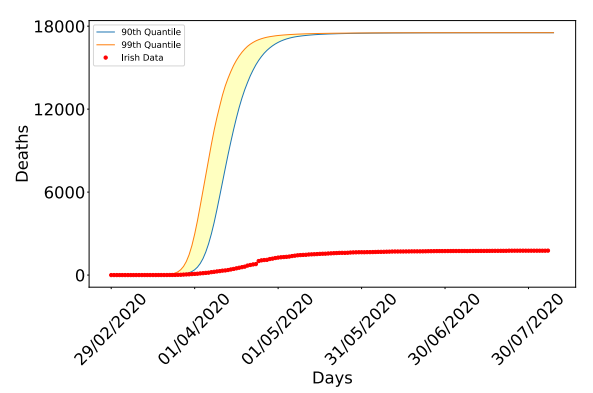

(b) Death Curve

FIG. 10. Infection and death curves produced by the model to simulate if no lockdown was implemented with social interaction at the same level as $t_{0}=0$ throughout the model.

clearly seen from Figure 10(a) that the rate of infection would be massively increased, as opposed to the population infection curve we have seen already in Figure 9(a) along with a much higher death curve shown in Figure 10(b) with predicted total deaths coming to roughly 17500 once again dwarfing the death curve seen in Figure 9(e). This indicates that some form of lockdown or policy intervention to reduce social contacts was warranted due to the potentially higher level of infections and deaths arising from no action being taken.

5.1.2. Earlier Lockdown. An earlier population-wide lockdown can be simulated by reducing the level of interaction within the population from that in period 3 
to the levels within period 2 as seen in Table 5 , such that $b_{i j}^{(2)}=b_{i j}^{(3)}$. The results of this analysis are shown in Figure 11 from which it can be seen to reduce the level of infection throughout the population with a significant reduction apparent in Cohort 3 as shown in Figure 11(d) due to the cocooning measures implemented during lockdown, which significantly reduced the social contacts of Cohort 3 for a greater portion of the model, effectively only giving members of Cohort 3 a period of 15 days from $t_{0}=0$ on February $29^{\text {th }}$ to contract the disease with their pre-lockdown level of interaction, thus reducing their likelihood of contraction. A population-wide reduction is also evident with the $75^{\text {th }}$ quantile of the models predicted range being reduced significantly in all cases indicating that the results in this scenario would be reduced infections. However a second spike scenario does appear to still be possible due to the evident resurgence towards the end of the modelling period, specifically in Cohort 1 as seen in Figure 11(b). Although on a population level the resurgence does appear to be less severe than that indicated in Section 9 due to the overall mass of infected people being reduced from the earlier lockdown.

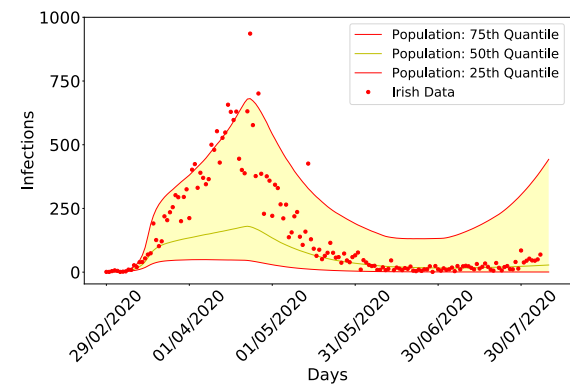

(a) Population

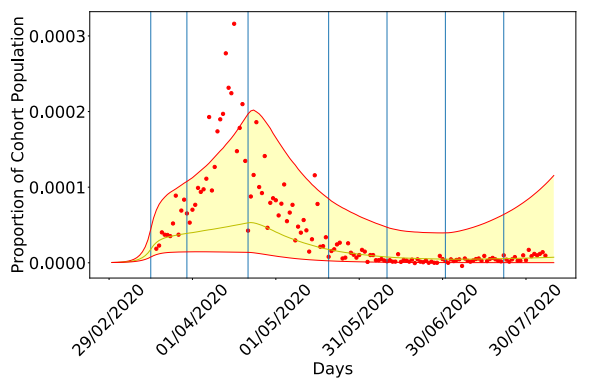

(c) Cohort 2 scaled to Cohort population

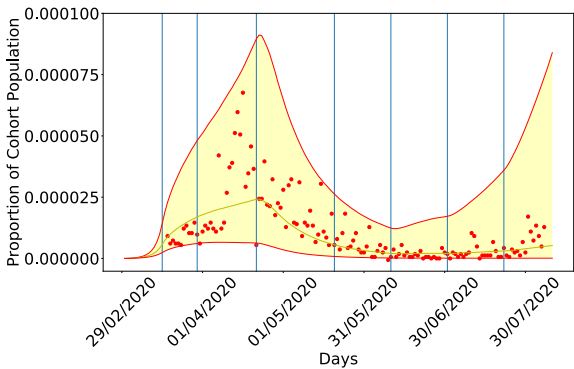

(b) Cohort 1 scaled to Cohort population

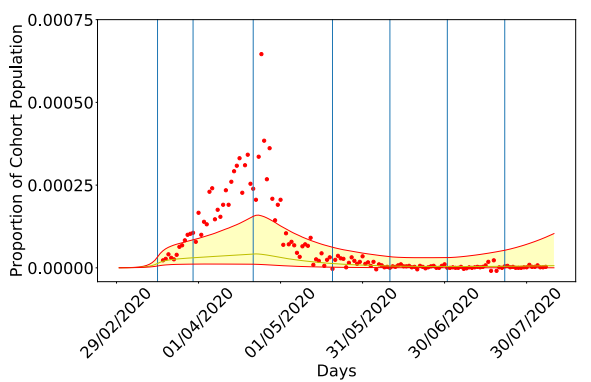

(d) Cohort 3 scaled to Cohort population

FIG. 11. A 50\% quantile range of infections if a lockdown was started from $t_{2}=15$ as opposed to the original start of lockdown from $t_{3}=27$.

5.1.3. Later School Closure. In the course of events leading up to the Irish lockdown, schools and universities were closed from Thursday $12^{\text {th }}$ March in period 2 of the model and remained closed throughout the modelling period. In order to simulate a later school closure the reduction in Cohort 1's level of interaction in period 2 has been delayed until the start of the full lockdown from period 3 onwards, as shown in Figure 5. In order to simulate this the level of interaction for period 2 including Cohort 1 as given by the constant values $b_{1 j}^{(2)}$ and $b_{i 1}^{(2)}$, seen in Table 5 
was set such that $b_{1 j}^{(2)}=b_{1 j}^{(1)}$ and $b_{i 1}^{(2)}=b_{i 1}^{(1)}$. The results of this can be seen in Figure 12, which along with the greatly increased level of infection in Cohort 1, as seen in Figure 12(a), would also result in an increase in infection for the rest of the population as seen in Figure 12(b) due to the higher level of aggregate social contact within the population. Due to the higher death rates seen in Section 4.1.7 and more adverse effects seen in the older members of the population[13] in Cohort 3, this would result in a much less favourable outcome for the majority of the population in this instance.

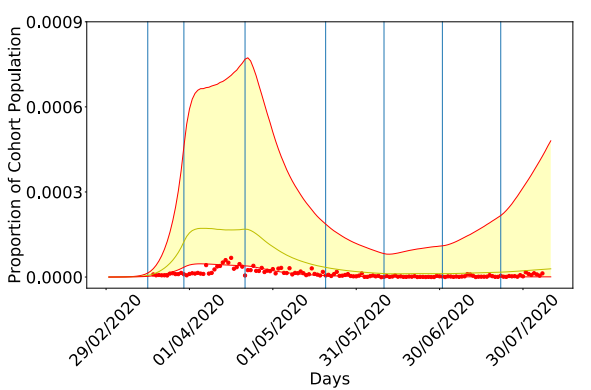

(a) Cohort 1

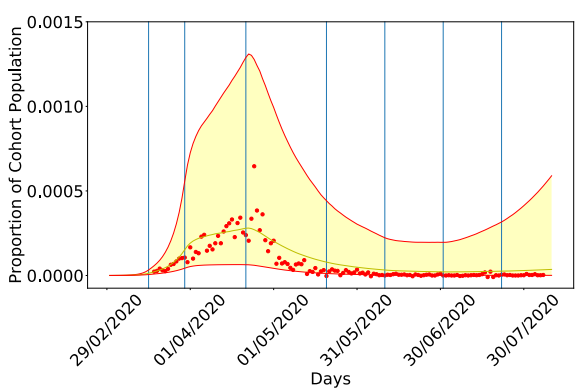

(b) Cohort 3

FIG. 12. Infection curve given under the assumption that school and university closure was delayed to $t_{3}=27$ as opposed to the original scenario when the closure occurred in period 2.

5.1.4. Partial Compliance. An important factor to consider when modelling the effect of policy is the level of compliance within a population to said policy. Throughout the past simulations we have assumed that the whole population complies with policy as reflected by varying levels of interaction seen in Table 5 . In order to simulate a proportion of non-compliant members of the population we will assume a level of non-compliance with policy given by $0 \leq \eta \leq 1$ such that a proportion of the population given by $\eta$ are assumed to interact at a rate $b_{i j}^{(0)}$ throughout the modelling period. Thus $\beta_{i j}(t)=\eta b_{i j}^{(0)}+(1-\eta) b_{i j}^{(\kappa)}$ for all $t \leq 160$. Here we assume a level of noncompliance $\eta=0.2$, meaning $20 \%$ of the population are assumed to interact at a constant rate $b_{i j}^{(0)}$ while $80 \%$ are compliant with policy and so interact according to the values established in Table 5. The results of this analysis can be seen in Figure 13 examining the population as a whole in terms of infections and deaths in Figures 13(a) and 13(b). Here we do not observe the suppression in infections caused by the second lockdown as seen in Figure 9 and instead see a much higher single curve representing the increased rate of infection and a higher level of total deaths of approximately 16000 under this framework. This highlights the overall importance of a large proportion of the population complying with policy to suppress the level of infection and deaths in the population.

5.2. Possible Future Scenarios. In this section we will generate projections relating to the time following the modelling period in order to evaluate an effective policy given the current position of the virus, directly following the modelling period based on the policies that have actually been implemented throughout this period. This will indicate if the country is ready for a further phase in its reopening plan or if this plan needs to be revised or possibly rolled back within the following projections. 


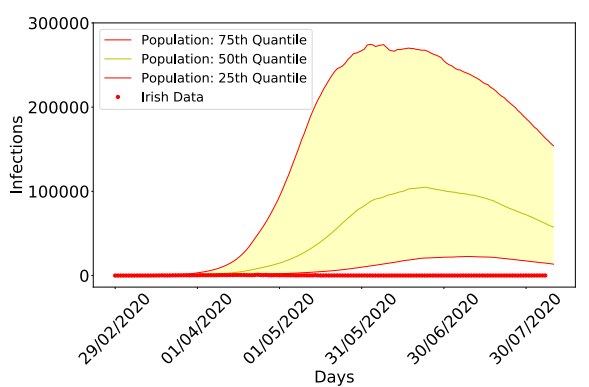

(a) Population Infections Curve

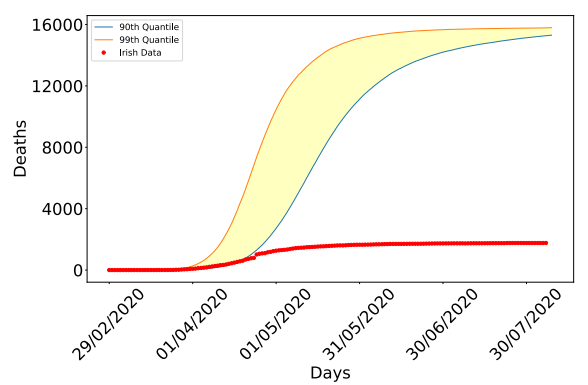

(b) Population Death Curve

FIG. 13. Compliance analysis with $\eta=0.2$ for $\beta_{i j}(t)=\eta b_{i j}^{(0)}+(1-\eta) b_{i j}^{(\kappa)}$ for all periods $0 \leq \kappa \leq 7$.

5.2.1. Current Course. As at the end of the modelling period the current course of the virus can be extended further by allowing the values of $\beta_{i j}\left(t^{*}\right)=\beta_{i j}\left(t_{8}\right)$ for all $t^{*} \geq 160$. This projection gives an idea of how the virus will progress at its current rate and if policy intervention will be required in the future. It can be seen in Figure 14 that the population in general would be much more adversely effected in terms of infections than was seen originally during the modelling period. It would have a peak of approximately 40000 infections dwarfing the original population peak of approximately 3500, as seen in Figure 9(a) which would lead to a much greater strain on the healthcare system than originally seen. This would indicate that policy

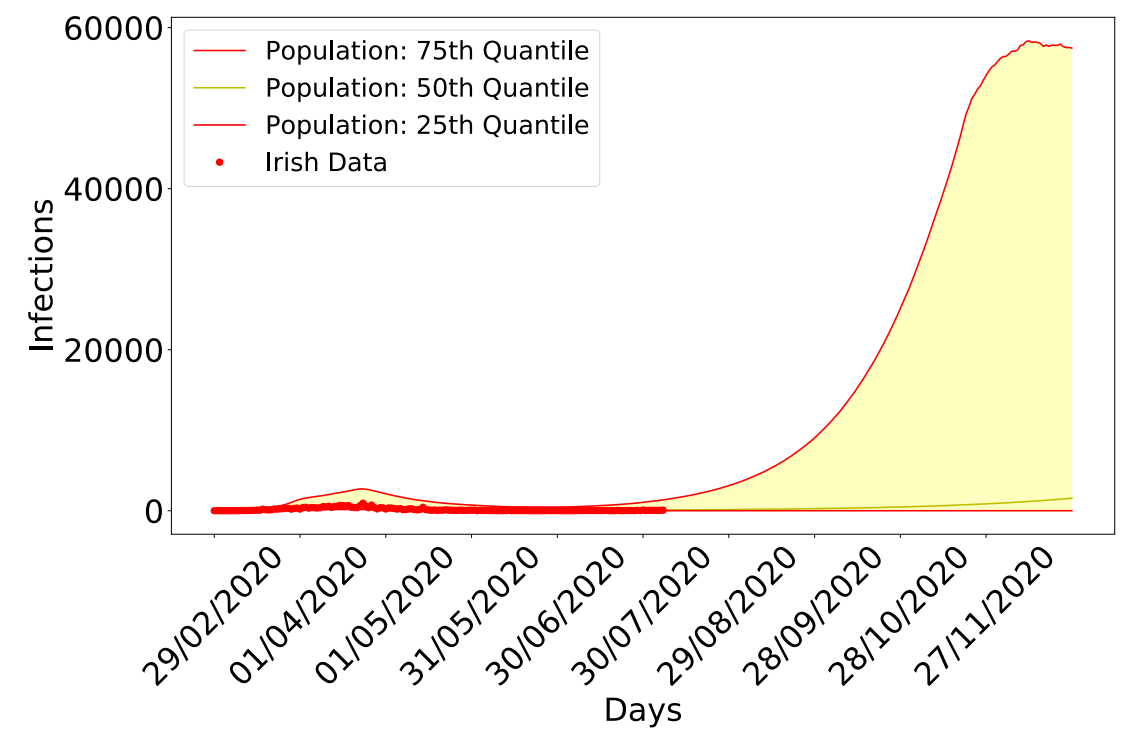

FIG. 14. Population-level projection until $t=300$ of infections with no change in the level of interaction from $t=160$.

intervention is required in order to reduce the future level of infection within the 
population. In terms of Cohort infections there is no discernible difference in growth between the relevant Cohorts over the examined time period. Some of the potential policy interventions will now be discussed below.

5.2.2. Reintroduce a Full Lockdown. A full lockdown projection gives a scenario such that a lockdown with the same level of social contact seen throughout the lockdown is reintroduced in order to mitigate the effects of the second spike evidenced in Section 5.2.1 in order to reduce the spread of the virus. This is achieved by setting the value of $\beta_{i j}\left(t^{*}\right)=\beta_{i j}\left(t_{3}\right)$ for all $t^{*} \geq 160$ such that the level of interaction is the same as that observed throughout the lockdown in the modelling period. The population level projection shown in Figure 15 indicates that the implemented lockdown would cause a turning point in the infection curve leading to a reduction towards 0 following the end of the projection as at $t=300$. From this point the lockdown policy could be revised given the situation at $t=300$ with consideration for economic and social factors.

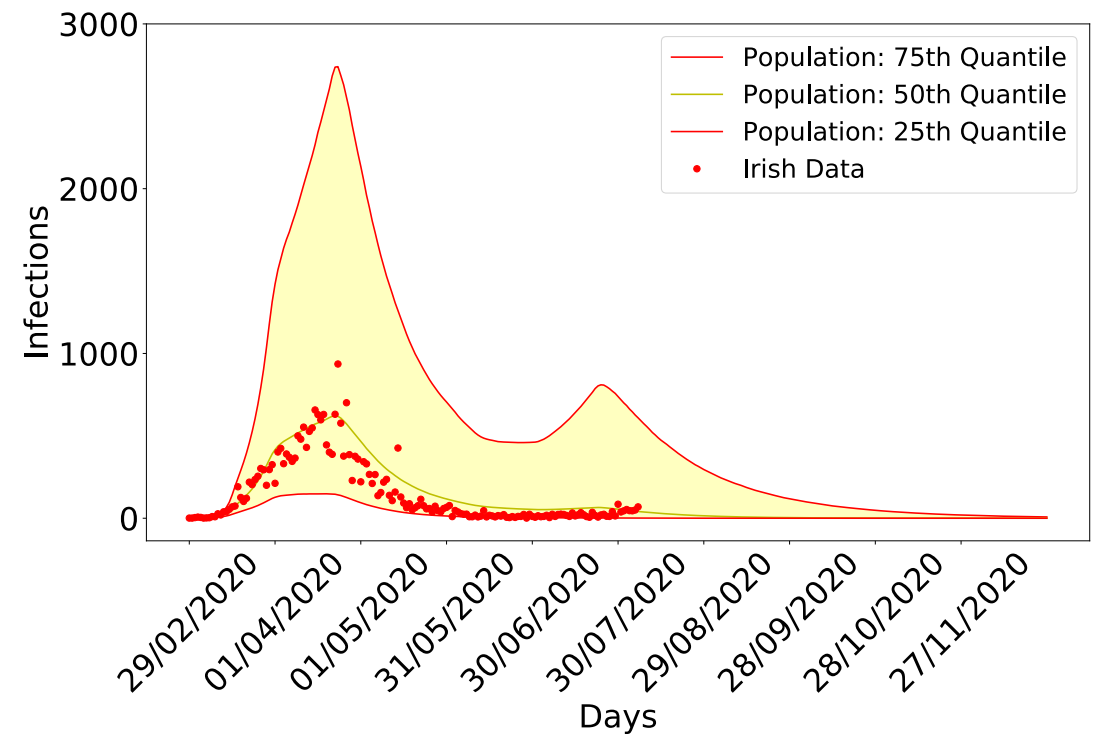

Fig. 15. Population level projection of infections under the assumption that a full lockdown is reimposed following the end of the modelling period until $t=300$.

5.2.3. Reintroduce Cocooning. An alternative policy to explore would be to cocoon the elderly again in order to shield the more vulnerable population within Cohort 3 as opposed to the full lockdown explored in Section 5.2.2. This is achieved by setting the value of $\beta_{i 3}\left(t^{*}\right)=\beta_{i 3}\left(t_{3}\right)$ and $\beta_{3 j}\left(t^{*}\right)=\beta_{3 j}\left(t_{3}\right)$ for all $t^{*} \geq 160$ such that any interaction involving Cohort 3 is reduced to the level seen throughout lockdown. Observing the results of this policy on the population, as seen in Figure 16, as opposed to the projections explored in Figure 14, with no new policies implemented it can be seen that while a second spike does appear to occur with this policy, the second spike occurs at a much more gradual rate over the population than infections originally grew at in the modelling period, with similar levels of growth seen seen across all Cohorts. This would indicate that the reintroduction of cocooning could be a viable 
option coupled with other measures that could be established later in the growth of infections due to the slow growth given by this policy allowing for a further reduction in infections.

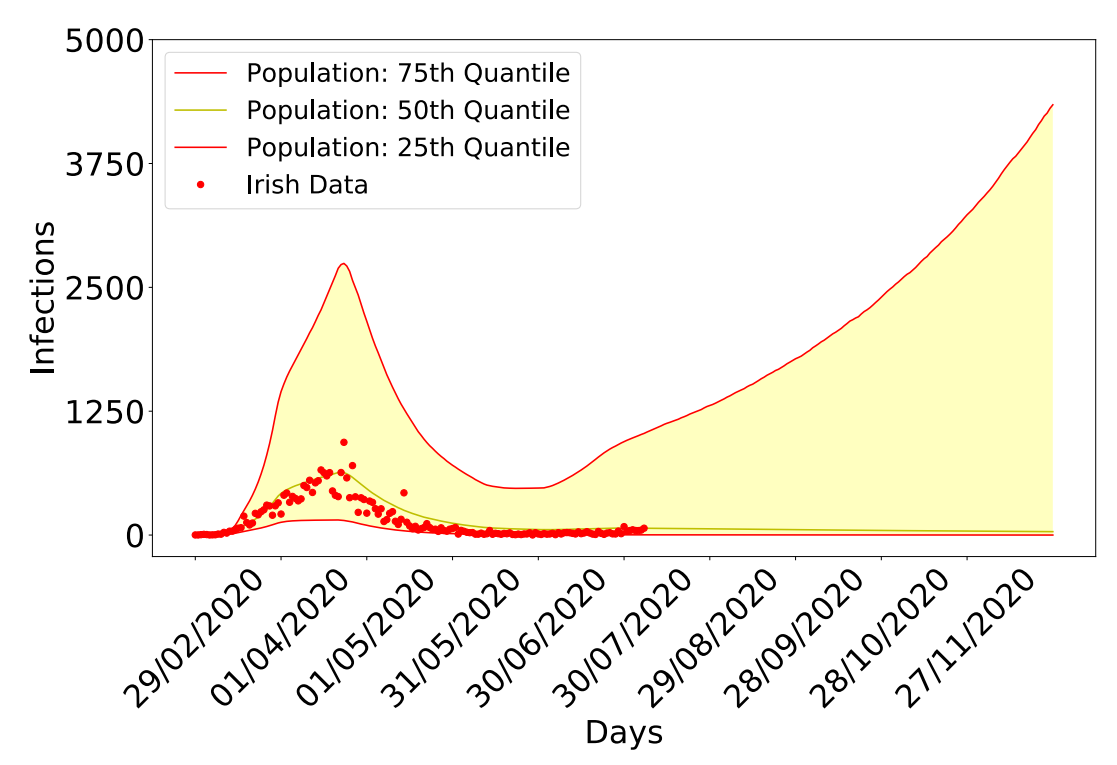

FIG. 16. Population-level projection of infections until $t=300$ with cocooning re-implemented.

6. Conclusion. From the projections and policy analysis carried out throughout Section 5 it is clear that any policy that reduces social contact between susceptible and infectious people within a population will reduce the growth of infections within the population as would be expected following examination of Equations (3.9) - (3.15). Hence, indicating that from the point of view of reducing infections to 0 a full lockdown policy such as that discussed in Sections 5.1.2 and 5.2.2 would be favourable as it appears to be the most effective approach in generating a disease-free equilibrium. However, if social freedom and economic factors are to be considered a more restrained policy such as reintroducing cocooning as discussed in Section 5.2.3 may be the most prudent strategy as it allows for a much slower growth and allows more time to implement further policies as the situation progresses while minimising the locked down population. It can also see from Section 5.1.4 that the impact of noncompliance with policy can be great and thus it is important that a the vast majority of the population complies with policy interventions as levels of non-compliance in the population can have detrimental effects on viral suppression.

The model does highlight the need for policy intervention and hinges on the fact that the population will adhere to newly implemented policies, so population wide adherence to policy is an important factor to consider in these results. Another issue highlighted within this model is the random nature of the spread of the virus, since biological parameters are implemented as random variables to illustrate varying physiologies from person to person, a range is produced as seen in the figures within Section 5 sometimes with a very wide potential range. These ranges reflect the fact that the spread of the virus is not inherently deterministic and in terms of expansion of this model, many other factors can be implemented as random variables to reflect 
this random characteristic of the spread of infections.

The modelling carried out represents a snapshot in time from February to August 2020 assuming one viral strain. The study of COVID-19 is an ongoing area of research that is rapidly developing. As of February $1^{\text {st }} 2021$ we know that COVID-19 has the capacity to present in a number of strains with varying levels of transmissibility and lethality. The existence of these strains could be examined as a potential area of further study in the future. Similarly the model could be extended over a longer period of time as more data becomes available.

Another area that could be developed on in further study is the fitting of the model to data using techniques such as least-squares regression, which has not been attempted here due to the stochastic nature of the model and high numbers of parameters. This could be achieved by considering the population model described in Equations (3.1) - (3.7) and focusing on either infection or death data which is freely available from Ordnance Survey Ireland's COVID Statistics Profile[15] as used in this model or from the WHO's Coronavirus Dashboard for analysis of countries other than Ireland[20]. It should be noted, however, that when considering data from the early stages of the pandemic that the full extent of infection may not be captured in the data. This could be due to reporting errors as aspects such as asymptomatic cases may have not been tested for or correctly identified in early stages due to a lack of familiarity with the disease at the time. Following this, Python libraries such as pandas and sklearn could be used to perform regression analysis and estimate alternative values for the interaction of mortality parameters $\beta$ and $\mu$, respectively.

Acknowledgements. I would like to acknowledge the support and advice given by Dr. Petri Piiroinen throughout the development of the model in supervising this project. Thanks are also extended for the financial support offered by the School of Mathematics, Statistics and Applied Mathematics at NUI Galway, as part of their Summer Internship Programme. I am also appreciative to the organisers of the $6^{\text {th }}$ Stokes Modelling Workshop at NUI Galway for facilitating the workshop, where I was able to develop the early stages of the model along with the team I worked with.

Appendix A. Calculation of the Basic Reproductive Number. To calculate the population-level basic reproductive number $R_{0}$ for a $k=3$ age-cohort SEIRD model described by Equations (3.9) - (3.15), we consider an aggregation of the 3 age-cohorts to represent the entire population such that,

$$
x_{1}=\sum_{i=1}^{3} S_{i}, \quad x_{2}=\sum_{i=1}^{3} E_{i}, \quad x_{3}=\sum_{i=1}^{3} I_{i}, \quad x_{4}=\sum_{i=1}^{3} R_{i}, \quad x_{5}=\sum_{i=1}^{3} D_{i} .
$$

It should be noted that in defining the compartment $x_{3}$ in Equation (A.1) we let $I_{i}=I_{a i}+I_{p i}+I_{s i}$ denote all actively infectious members of cohort $i$, which we will use below for brevity. From this we can identify the relevant dynamic variables $x_{2}$ and $x_{3}$ and express them as the vector,

$$
x=\left(x_{2}, x_{3}\right)
$$

Next, we consider the matrices $F$ and $V$ about a disease free equilibrium point such that $x=(0,0)$ and compute the next-generation operator $F V^{-1}$ as described in 
Section 3.3. The matrices are

$$
\begin{aligned}
& F=\frac{\partial F_{i}\left(x_{0}\right)}{\partial x_{j}}=\left(\begin{array}{cc}
0 & \sum_{j} \sum_{i} \beta_{i j} \\
0 & 0
\end{array}\right), \\
& V=\frac{\partial V_{i}\left(x_{0}\right)}{\partial x_{j}}=\left(\begin{array}{cc}
\sigma & 0 \\
-\sigma & \sum_{i} \gamma_{i}
\end{array}\right), \\
& F V^{-1}=\left(\begin{array}{cc}
\frac{\sum_{j} \sum_{i} \beta_{i j}}{\sum_{i} \gamma_{i}} & \frac{\sum_{j} \sum_{i} \beta_{i j}}{\sum_{i} \gamma_{i}} \\
0 & 0
\end{array}\right) .
\end{aligned}
$$

The basic reproductive number is computed by taking the eigenvalues $\lambda_{i}$ of this matrix and assigning the leading eigenvalue as $R_{0}$, such that

$$
\begin{aligned}
& \lambda_{1}, \lambda_{2}=0, \frac{\sum_{j} \sum_{i} \beta_{i j}}{\sum_{i} \gamma_{i}}, \\
& R_{0}=\operatorname{Max}\left(\lambda_{1}, \lambda_{2}\right), \\
& \Longrightarrow R_{0}=\frac{\sum_{j} \sum_{i} \beta_{i j}}{\sum_{i} \gamma_{i}},
\end{aligned}
$$

as seen in Equation (3.19).

\section{REFERENCES}

[1] Arcede, Jayrold P., Caga-anan, Randy L., Mentuda, Cheryl Q., and Mammeri, Youcef, Accounting for Symptomatic and Asymptomatic in a SEIR-type model of COVID-19, Math. Model. Nat. Phenom., 15 (2020), p. 34, https://doi.org/10.1051/mmnp/2020021.

[2] D. Buitrago-Garcia, D. Egli-Gany, M. J. Counotte, S. Hossmann, H. Imeri, A. M. IPEKCI, G. SAlanti, AND N. LOW, Occurrence and transmission potential of asymptomatic and presymptomatic SARS-CoV-2 infections: A living systematic review and metaanalysis, PLOS Medicine, 17 (2020), pp. 1-25, https://doi.org/10.1371/journal.pmed. 1003346.

[3] Central Statistics Office, E3001: Enumerated Population 1926 to 2016 by Age Group, Sex and Census Year, 2016, https://statbank.cso.ie/px/pxeirestat/Statire/SelectVarVal/ saveselections.asp\#. Accessed 8/7/2020.

[4] M. Cevik, J. L. Marcus, C. Buckee, and T. C. Smith, Severe Acute Respiratory Syndrome Coronavirus 2 (SARS-CoV-2) Transmission Dynamics Should Inform Policy, Clinical Infectious Diseases, (2020), https://doi.org/10.1093/cid/ciaa1442.

[5] A. DAS, An approximation-based approach for periodic estimation of effective reproduction number: a tool for decision-making in the context of coronavirus disease 2019 (COVID19) outbreak, Public Health, 185 (2020), pp. 199 - 201, https://doi.org/10.1016/j.puhe. 2020.06.047.

[6] S. Flaxman, S. Mishra, A. Gandy, H. Unwin, H. Coupland, T. Mellan, H. Zhu, T. Berah, J. Eaton, P. Perez Guzman, N. Schmit, L. Cilloni, K. Ainslie, M. Baguelin, I. Blake, A. Boonyasiri, O. Boyd, L. Cattarino, C. Ciavarella, L. Cooper, Z. Cucunuba Perez, G. Cuomo-Dannenburg, A. Dighe, A. Djaafara, i. Dorigatti, S. Van Elshand, R. Fitzjohn, H. Fu, K. Gaythorpe, L. Geidelberg, N. Grassly, W. Green, T. Hallett, A. Hamlet, W. Hinsley, B. Jeffrey, D. Jorgensen, E. Knock, D. Laydon, G. Nedjati Gilani, P. Nouvellet, K. Parag, I. Siveroni, H. Thompson, R. Verity, E. Volz, C. Walters, H. Wang, Y. Wang, O. Watson, P. Winskill, X. Xi, C. Whittaker, P. Walker, A. Ghani, C. Donnelly, S. Riley, L. Okell, M. Vollmer, N. Ferguson, and S. Bhatt, Report 13: Estimating the number of infections and the impact of non-pharmaceutical interventions on COVID-19 in 11 European countries, tech. report, Imperial College London, 2020, https://doi.org/10.25561/77731.

[7] J. Heffernan, R. Smith, And L. Wahl, Perspectives on the basic reproductive ratio, Journal of The Royal Society Interface, 2 (2005), pp. 281-293, https://doi.org/10.1098/rsif.2005.0042. 
[8] HPSC, Epidemiology of COVID-19 in Ireland, https://www.hpsc.ie/a-z/respiratory/ coronavirus/novelcoronavirus/casesinireland/epidemiologyofcovid-19inireland/. Accessed $3 / 8 / 2020$.

[9] IEMAG Epidemiology Modelling Subgroup, A population-level SEIR model for COVID-19 scenarios, Department of Health, (2020).

[10] IEMAG Epidemiology Modelling subgroup, COVID-19 epidemiological parameters summary document, Department of Health, (2020).

[11] IEMAG Epidemiology Modelling subgroup, Estimation of the Effective Reproductive Num$\operatorname{ber}\left(R_{t}\right), 2020$.

[12] W. O. Kermack, A. G. MCKendrick, and G. T. Walker, A contribution to the mathematical theory of epidemics, Proceedings of the Royal Society of London. Series A, Containing Papers of a Mathematical and Physical Character, 115 (1927), pp. 700-721, https://doi. org/10.1098/rspa.1927.0118.

[13] K. Liu, Y. Chen, R. Lin, And K. Han, Clinical features of COVID-19 in elderly patients: A comparison with young and middle-aged patients, Journal of Infection, 80 (2020), pp. e14 - e18, https://doi.org/10.1016/j.jinf.2020.03.005.

[14] NPHET, Minutes and Agendas from meetings of the National Public Health Emergency Team: COVID-19 (Coronavirus), 2020.

[15] Ordnance Survey Ireland, COVID Statistics Profile HPSC Ireland Open Data, data.gov.ie/ dataset/covidstatisticsprofilehpscirelandopendata1. Accessed 8/8/2020.

[16] S. Perlman, Another decade, another coronavirus, New England Journal of Medicine, 382 (2020), pp. 760-762, https://doi.org/10.1056/NEJMe2001126. PMID: 31978944.

[17] E. M. Rees, E. S. Nightingale, Y. Jafari, N. R. Waterlow, S. Clifford, C. A. B. Pearson, C. W. Group, T. Jombart, S. R. Procter, and G. M. Knight, COVID-19 length of hospital stay: a systematic review and data synthesis, BMC Medicine, 18 (2020), p. 270, https://doi.org/10.1186/s12916-020-01726-3.

[18] G. Van Rossum and F. L. Drake, Python 3 Reference Manual, CreateSpace, Scotts Valley, CA, 2009, https://www.python.org/.

[19] World Health Organisation, Q $\& A$ on Coronaviruses (COVID-19), https: //www.who.int/emergencies/diseases/novel-coronavirus-2019/question-and-answers-hub/ q-a-detail/q-a-coronaviruses. Accessed 30/7/2020.

[20] World Health Organisation, WHO Coronavirus (COVID-19) Dashboard, covid19.who.int. Accessed 30/03/2021 